\title{
Quasi-Coherent Noise Jamming to LFM Radar Based on Pseudo-Random Sequence Phase-modulation
}

\author{
Ning TAI, Yujian PAN, Naichang YUAN \\ College of Electronic Science and Engineering, National University of Defense Technology, \\ Changsha, Hunan, 410073, China \\ 358041578@qq.com
}

\begin{abstract}
A novel quasi-coherent noise jamming method is proposed against linear frequency modulation (LFM) signal and pulse compression radar. Based on the structure of digital radio frequency memory (DRFM), the jamming signal is acquired by the pseudo-random sequence phase-modulation of sampled radar signal. The characteristic of jamming signal in time domain and frequency domain is analyzed in detail. Results of ambiguity function indicate that the blanket jamming effect along the range direction will be formed when jamming signal passes through the matched filter. By flexible controlling the parameters of interrupted-sampling pulse and pseudo-random sequence, different covering distances and jamming effects will be achieved. When the jamming power is equivalent, this jamming obtains higher process gain compared with non-coherent jamming. The jamming signal enhances the detection threshold and the real target avoids being detected. Simulation results and circuit engineering implementation validate that the jamming signal covers real target effectively.
\end{abstract}

\section{Keywords}

LFM, electronic countermeasure, DRFM, pseudorandom sequence, interrupted-sampling

\section{Introduction}

Modern radar usually uses wave-coherence to implement pulse accumulation, which significantly depresses the non-coherent jamming and puts forward great challenge to jammer. LFM signal is widely applied because of the characteristic of large band-time product. By pulse compression, LFM signal can achieve high resolution and detecting distance [1-4].

The popular jamming method against LFM radar is based on DRFM to duplicate radar signal without distortion. The modulated sampled signal is transmitted to victim radar after some time delay for creating coherent jamming. But the produced false-target in range direction is behind the real target $[5,6,7]$. By using the property of matched filter, shift- frequency jamming attaches a value of shift-frequency to radar signal to achieve the precede jamming or hysteretic jamming. Taking the mismatch impact of matched filter into consideration, the value of shift-frequency shall not exceed the bandwidth of mated filter. The jamming signal with a large value of shift-frequency can't obtain good jamming effect $[8,9,10]$. When radar adopts chirp-rate-agility or frequencydiversity of different chirp rate, the above false-targets appear in different range cell and can be recognized correctly. Thus the false-targets produced by original shift-frequency are restrained after several pulses accumulation. According to the features that the frequency difference of LFM signal is independent on time, method of shift-frequency jamming based on delay invariance is proposed $[11,12]$. By controlling the frequency shift amount, this jamming method can produce the false-target of the same range distance.

Interrupted-sampling method solves the problem of high isolation between receiving antenna and transmitting antenna. The jamming signal is obtained by intermit sampling, storing and transmitting radar signal. Mathematic analysis indicates that this jamming signal can form multiple false-targets along the range direction. But these false-targets are distributed symmetrically and of which the amplitudes are not enough $[13,14]$. Because the period and duty ratio of interrupted-sampling pulse affects the distribution characteristic of false-targets, method of interrupted-sampling and periodic repeater is proposed. After sampling a segment of radar signal, jammer re-transmits this signal periodically until the end the sampling period, which enhances the jamming duty factor and produces multiple false-targets of strong amplitudes [15].

Radar echo cancellation idea is proposed in [16], of which the main method is using interrupted-sampling repeater to produce precede false target. The false target synchronizes with target echo in time domain but their phases are opposite. This feature is applied in target stealth technology and prevents target from being detected. And this method is expanded to 2-D for countering synthetic aperture radar (SAR) in [17], where a novel three-stage active cancellation method using frequency and delay-time modulation is demonstrated. The cancellation echo intentionally lags behind the target echo for the extra processing time when SAR transmitting LFM signal. 
High resolution imaging radars, such as SAR and inverse synthetic aperture radar (ISAR), use coherent process both in range-direction and azimuth-direction to obtain high quality 2-D image. The delayed jamming against bi- and multistatic SAR systems is proposed in [18]. Unlike conventional repeater jamming, the delay time of this jammer is random in the whole range interval and the jamming signal is transmitted to the scene with target intended to be protected. Due to the random delay time in the synthetic aperture time, the random phase term appears in the re-transmitted jamming signal and this phase causes mismatch in azimuth processing.

Pace P. E. et.al $[19,20]$ propose the idea of digital image synthesizer (DIS) in 2002, which is a deceptive jamming method for countering ISAR. It is implemented through coefficient modulation extracted from existing template and the corresponding false image of template is induced after imaging process. Considering the above modulation is somewhat complicated and with large computational complexity, Xu proposes two-stage DIS (T-DIS) [21], of which the modulation process is divided into the offline stage and the realtime stage. The improved structure represents simplicity and flexibility, thus reducing the computation time and hardware constraints. Knowing that DIS method emulates target based on point models, Zhao proposes a method combing the translation modulation with the electromagnetic model to achieve more verisimilar false target [22]. By utilizing the electromagnetic scattering model, the false target contains the structural information, such as multiple scattering and shadowing.

The researches in latest years show that false target deception jamming requires modulation process of high complexity and tremendous computation, thus noise jamming is developed and widely applied for its effectiveness and convenience. Noise jamming method is that jammer transmits noise signal or noise-like jamming signal to victim radar, which decreases the signal-to-noise ratio and prevents radar from detecting real target $[23,24]$. The common noise signal contains radio frequency noise, noise amplitude modulation jamming and noise phase modulation jamming. These noise jamming signals don't need too much information about the hostile radar and they are easy to be implemented. Because of the non-coherence with radar, these jamming signals need large power to achieve good jamming effect.

Aiming at obtaining process gain, smart noise is an effective method for countering coherent radar, which is able to produce dense multiple false-targets by multiplication modulation [25]. This jamming method contains the properties of noise signal and it covers target echo both in time domain and frequency domain. Thus it can both achieve blanket jamming and deception jamming according to the different modulation coefficient. The smart noise jamming is developed in [26], where a Gauss white noise frequency modulated signal is introduced. This jamming signal is almost the same as target echo in time domain and frequency domain, which brings difficult for radar to distinguish the jamming signal. Accord- ing to the bandwidth of Gauss white noise, jamming signal flexibly produces blanket jamming or deception jamming.

While both the multiplication modulation and frequency modulation method is based on the whole radar signal and the modulation unit consumes large resources, our purpose is to design a jammer capable of producing effective noise, with simple structure and low cost. Thus in this paper we propose a pseudo-random sequence phase-modulation jamming signal based on DRFM structure. This jamming signal is created through phase-modulation on radar signal, thus it has much correlation with matched filter. Because phase-modulation by pseudo-random sequence spreads the bandwidth of radar signal and wreaks the coherence between jamming signal and reference signal, jamming signal forms the noise-like signal that covers some frequency range. Compared with other non-coherent noise jamming or convolution jamming signal, our proposed jamming method is easy to be implemented and with simpler structure. Simulation results show that it can effectively jam the hostile radar and save jamming power.

\section{Jamming Signal Model}

\subsection{Interrupted-Sampling}

This section briefly introduces the theory of interruptedsampling and direct transmitting jamming. The basic procedure is as follows: A periodic pulse train is used to sample the radar signal and the sampled signal is transmitted between two sampling period.

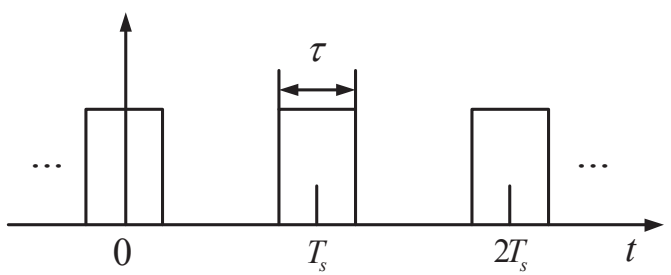

Fig. 1. Interrupted-sampling pulse.

Figure 1 shows the interrupted-sampling pulse $p(t)$, of which the envelope is a rectangular pulse train with pulsewidth defined as $\tau$, sampling period as $T_{s} . \operatorname{rect}(t / \tau)=1$ when $0<t<\tau$, otherwise it is 0

$$
p(t)=\operatorname{rect}\left(\frac{t}{\tau}\right) * \sum_{n=-\infty}^{+\infty} \delta\left(t-n T_{s}\right),
$$

of which the spectrum is

$$
P(f)=\sum_{n=-\infty}^{+\infty} \tau f_{s} \times \operatorname{sa}\left(\pi n f_{s} \tau\right) \times \delta\left(f-n f_{s}\right),
$$

where $\operatorname{sa}(x)=\sin (x) / x, f_{s}=1 / T_{s}$ means the sampling frequency. On the occasion $T_{s}=2 \tau, p(t)$ is a square pulse train, and (2) becomes 


$$
P(f)=\sum_{n=-\infty}^{+\infty} \frac{1}{2} \times \operatorname{sa}\left(\frac{n \pi}{2}\right) \times \delta\left(f-n f_{s}\right) .
$$

When $n$ is even except for zero, the amplitude of (3) is zero. The amplitude modulated by $s a(\cdot)$ function decreases with the increasing of level $n$.

\subsection{Phase-Modulation}

Pseudo-random sequence is widely applied in spreadspectrum communication. The sequence can be created by a shift-register. The expression of pseudo-random sequence in one period in time domain can be defined as

$$
\begin{aligned}
u(t) & =\operatorname{rect}\left(\frac{t}{T_{c}}\right) * \sum_{m=0}^{N-1} c_{m} \times \delta\left(t-m T_{c}\right) \\
& =u_{1}(t) * u_{2}(t)
\end{aligned}
$$

where $c_{m}= \pm 1, T_{c}$ is code width and $*$ is convolution. $u_{1}(t)$ is the child pulse signal with pulse width of $T_{c} \cdot u_{2}(t)$ is the Dirac impulse function containing the sequence amplitude $c_{m}$. For a shift-register of level $n$, the sequence period $P$ equals to $2^{n}-1$

$$
\begin{gathered}
U_{1}(f)=T_{c} \times \operatorname{sinc}\left(f T_{c}\right), \\
U_{2}(f)=\sum_{m=0}^{P-1} c_{m} \times \exp \left(-j 2 \pi f m T_{c}\right) .
\end{gathered}
$$

Applying spectrum of $u_{1}(t)$ and $u_{2}(t)$ in (5) (6), we obtain the spectrum of pseudo-random sequence

$$
U(f)=T_{c} \times \operatorname{sinc}\left(\pi f T_{c}\right) \times \sum_{m=0}^{P-1} c_{m} \times \exp (-j 2 \pi f m T)
$$

Computing result indicates that the bandwidth of pseudo-random sequence approximates to the bandwidth of child pulse $u_{1}(t)$

$$
B_{w}=\frac{1}{T_{c}}
$$

To further analyze the characteristic of pseudo-random sequence, the power spectrum is given in (9)

$$
G(f)=\frac{P+1}{P^{2}}\left(\frac{\sin \left(\pi f T_{c}\right)}{\pi f T_{c}}\right)^{2} \sum_{\substack{l=-\infty \\ l \neq 0}}^{+\infty} \delta\left(f-\frac{l}{P T_{c}}\right)+\frac{1}{P^{2}} \delta(f) .
$$

From (9) we can see that the power spectrum is discrete line spectrum and the frequency distance between two neighboring spectral line is $1 / P T_{c}$. The amplitude of spectral line is determined by sinc function. The amplitude is 0 when the spectral line level $l$ is the multiple integer of $P$.

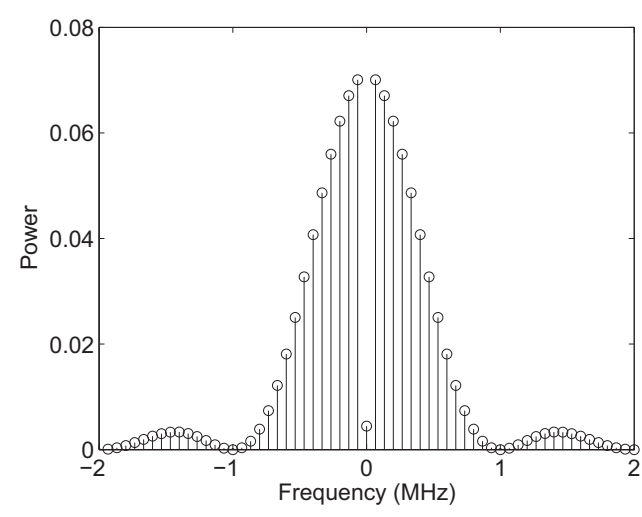

Fig. 2. Power spectrum of pseudo-random sequence.

Figure 2 shows the power spectrum when $P=15$, $T_{c}=1 \mu \mathrm{s}$. Because pseudo-random sequence is with constant power, mathematic computation shows that $90 \%$ power of the sequence is located in $\left[-1 / T_{c},+1 / T_{c}\right]$. Interval $\left[-1 / T_{c},+1 / T_{c}\right]$ becomes large when $T_{c}$ gets small. But the amplitude of spectral line decreases.

\subsection{Jamming Signal}

Assume that the radar signal is $s(t)$, of which the spectrum is $S(f)$. The creation of jamming signal is as follows: interrupted-sampling pulse $p(t)$ is used to sample the radar signal $s(t)$, the transmitted sampled signal is modulated by pseudo-random sequence $u(t)$. The jamming signal equals to the multiplication of $s(t), u(t)$ and $p(t)$

$$
s_{j}(t)=s(t) \times u(t) \times p(t) .
$$

Assume that radar signal transmits LFM signal. The pulse width of LFM signal is $T$ and chirp rate is $k$. $s(t)$ reaches the location of jammer after transmitting delay $T_{t}$. $\tau_{d}$ is the transmitting delay of jammer. Thus the sampled radar signal can be treated as a superposition of $N$ segments radar signal. $N$ is $T / T_{s}$ when $T / T_{S}$ is integer, otherwise is $\left[T / T_{s}\right]+1$, in which $[\cdot]$ means rounding numbers.

The $n^{\text {th }}$ segment sampled radar signal can be written as below

$$
\begin{aligned}
s_{n}(t)= & \operatorname{rect}\left(\frac{t-T_{t}-(n-1) T_{s}}{\tau}\right) \times \exp \left\{j 2 \pi \left[f_{0}(t-\right.\right. \\
& \left.\left.\left.T_{t}\right)+\frac{1}{2} k\left(t-T_{t}\right)^{2}\right]\right\} .
\end{aligned}
$$

And the $n^{\text {th }}$ transmitted jamming signal can be described as follows

$$
\begin{aligned}
s_{j n}(t)= & \operatorname{rect}\left(\frac{t-T_{t}-\tau_{d}-(n-1) T_{s}}{\tau}\right) \times \exp \left\{j 2 \pi \left[f_{0}(t-\right.\right. \\
& \left.\left.\left.T_{t}-\tau_{d}\right)+\frac{1}{2} k\left(t-T_{t}-\tau_{d}\right)^{2}\right]\right\} \times u_{1}(n) .
\end{aligned}
$$

$u_{1}(n)$ is the part of pseudo-random sequence which locates in the transmitting window of jammer. In order to maintain the phase-modulation on radar signal, code width should satisfy the condition $T_{c}<\tau$. From (12) we can see that the pulse 
width of $s_{j n}(t)$ is $\tau$ (For simplicity, we consider the situation that during the transmitting window only one sampled radar signal is transmitted). During the pulse width $\tau$, the original radar signal is modulated by part of pseudo-random serial $u_{1}(n)$. The value of jamming signal equals to the original value or the opposite value of radar signal during code with $T_{c}$. When the neighboring two code values are the same, the phase of jamming signal is consistent, otherwise there is a phase jump of $\pi$. This dis-consistent in phase spreads the bandwidth of modulated signal.

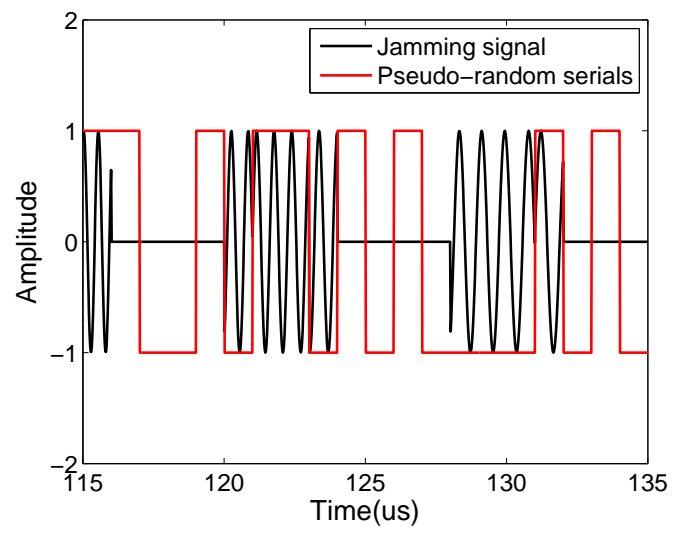

Fig. 3. Jamming signal and pseudo-random sequence.

Part of the jamming signal and pseudo-random sequence is shown in Fig. 3. In the simulation, the period of interrupted-sampling pulse is $8 \mu$ s with a duty ratio of $50 \%$. The length of pseudo-random sequence is 511 and code width is $0.5 \mu \mathrm{s}$. From Fig. 3 we can see that when the value of pseudo-random sequence changes, there will be a phase jump in the phase of jamming signal.

According to the theory of signal processing, the spectrum of jamming signal $s_{j}(t)$ is shown in (13)

$$
S_{J}(f)=S(f) * G(f) * P(f) .
$$

For simplicity, we use the power spectrum of pseudorandom sequence to analyze the characteristic of jamming signal. Substituting (2) and (9) into (13), we arrive at (14). The situation when $T_{s}=2 \tau$ is easy to control and implement. And (14) becomes (15).

In $(15), a_{n}=\frac{1}{2 P^{2}} \mathrm{sa}\left(\frac{n \pi}{2}\right), \quad b_{n l}=\frac{P+1}{2 P^{2}} \mathrm{sa}\left(\frac{n \pi}{2}\right) \mathrm{sa}^{2}\left(\frac{l \pi}{P}\right)$. Equation (15) shows that $S_{J}(f)$ is a superposition of weighted shift-frequency replicas of $S(f)$. The shift-frequency signal consists of two parts. One part is the symmetrically distributed $S\left(f-n f_{s}\right)$ created by interrupted-sampling. The distance of $S\left(f-n f_{s}\right)$ in frequency is determined by the sampling frequency of interrupted-sampling pulse. The other part is the small shift-frequency $S\left(f-n f_{s}-l / P T_{c}\right)$ around $S\left(f-n f_{s}\right)$. The shift-frequency amount of this part is determined by the characteristic of pseudo-random sequence.

The amplitude of $S\left(f-n f_{s}\right)$ is modulated by $s a$ function. When $n$ is $0, a_{n}=1 / 2 P^{2}$. When $n$ is odd, $a_{n}= \pm 1 / n \pi P^{2}$, otherwise $a_{n}=0$. When $P$ is fixed, $\left|a_{n}\right|$ is inversely proportional to $n$. The amplitude characteristic of $S\left(f-n f_{s}-l / P T_{c}\right)$ is similar to that of $S\left(f-n f_{s}\right)$. And the amplitude of $S\left(f-n f_{s}-l / P T_{c}\right)$ is 0 when $l$ is the integer multiples of $P$.

The spectrums of radar signal and jamming signal are shown in Fig. 4 and Fig. 5 respectively. In the simulation, the bandwidth of LFM signal is $5 \mathrm{MHz}$ and code width of pseudo-random sequence is $1 \mu \mathrm{s}$. From the figures we can see the spectrum of jamming signal is further spread compared with spectrum of LFM signal. The linearity character between time and frequency is wrecked. The amplitude of spectral line decreases with the frequency moving away from center frequency.

\section{Matched Filter Result}

The matched filter of radar signal $s(t)$ is $h(t)=s^{*}(-t)$, of which the spectrum is

$$
H(f)=S^{*}(f) .
$$

$$
\begin{aligned}
S_{J}(f) & =\sum_{n=-\infty}^{\infty} \tau f_{s} s a\left(\pi n f_{s} \tau\right) S\left(f-n f_{s}\right) * \frac{P+1}{P^{2}}\left(\frac{\sin \left(\pi f T_{c}\right)}{\pi f T_{c}}\right)^{2} \sum_{\substack{l=-\infty \\
l \neq 0}}^{+\infty} \delta\left(f-\frac{l}{P T_{c}}\right)+\frac{1}{P^{2}} \delta(f) \\
& =\sum_{n=-\infty}^{\infty} \frac{\tau f_{s}}{P^{2}} s a\left(\pi n f_{s} \tau\right) S\left(f-n f_{s}\right)+\sum_{n=-\infty}^{\infty} \sum_{\substack{l=-\infty \\
l \neq 0}}^{+\infty} \frac{\tau f_{s}(P+1)}{P^{2}} s a\left(\pi n f_{s} \tau\right) s a^{2}\left(\frac{l \pi}{P}\right) S\left(f-n f_{s}-\frac{l}{P T_{c}}\right) \\
S_{J}(f) & =\sum_{n=-\infty}^{\infty} \frac{1}{2 P^{2}} s a\left(\frac{n \pi}{2}\right) S\left(f-n f_{S}\right)+\sum_{n=-\infty}^{\infty} \sum_{\substack{l=-\infty \\
l \neq 0}}^{+\infty} \frac{P+1}{2 P^{2}} s a\left(\frac{n \pi}{2}\right) s a^{2}\left(\frac{l \pi}{P}\right) S\left(f-n f_{s}-\frac{l}{P T_{c}}\right) \\
= & a_{n} \times S\left(f-n f_{s}\right)+\sum_{n=-\infty}^{\infty} \sum_{\substack{l=-\infty \\
l \neq 0}}^{+\infty} b_{n l} \times S\left(f-n f_{s}-\frac{l}{P T_{c}}\right) .
\end{aligned}
$$




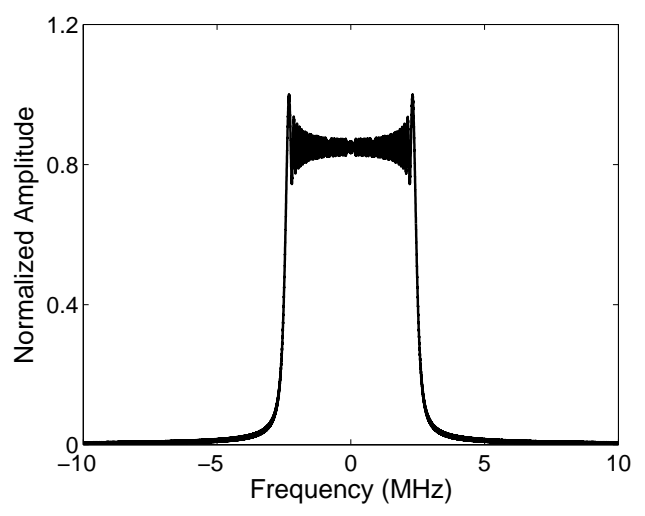

Fig. 4. Spectrum of LFM signal.

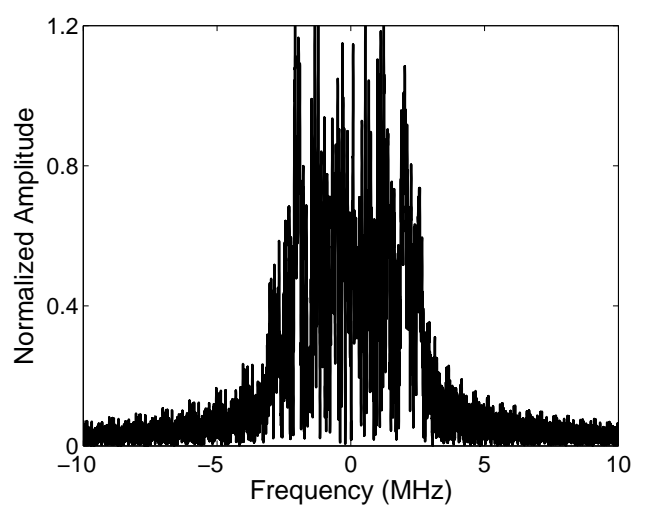

Fig. 5. Spectrum of jamming signal.

The processing procedure of jamming signal after matched filter is as follows:

$$
y_{j}(t)=s_{j}(t) * h(t) .
$$

The spectrum of output signal is

$$
Y_{J}(f)=S_{J}(f) H(f) .
$$

Substituting (15) and (16) into (18), we derive

$$
\begin{aligned}
Y_{J}(f)= & {\left[\sum_{n=-\infty}^{\infty} a_{n} \times S\left(f-n f_{s}\right)+\right.} \\
& \left.\sum_{n=-\infty}^{\infty} \sum_{\substack{l=-\infty \\
l \neq 0}}^{+\infty} b_{n l} \times S\left(f-n f_{s}-\frac{l}{P T_{c}}\right)\right] S^{*}(f) .
\end{aligned}
$$

The characteristic of $y_{j}(t)$ can be analyzed using ambiguity function. The ambiguity function of radar signal is defined as below

$$
\chi(\tau, \xi)=\int_{-\infty}^{+\infty} s(t) s^{*}(t-\tau) e^{-j 2 \pi \xi t} d t .
$$

Substituting $h(t)=s^{*}(-t)$ into (20), we arrive at

$$
\begin{aligned}
\chi(\tau, \xi) & =\int_{-\infty}^{+\infty}\left[s(t) e^{-j 2 \pi \xi t}\right] s^{*}(t-\tau) d t \\
& =\int_{-\infty}^{+\infty} w(t) h(t-\tau) d t \\
& =w(\tau) * h(\tau),
\end{aligned}
$$

where $w(t)=s(t) e^{-j 2 \pi \xi t}$ represents the target echo of a Doppler frequency shift $f_{d}=-\xi$ and $\chi(\tau, \xi)$ can be treated as the output of matched filter.

From (19) we know that $S\left(f-n f_{s}\right) S^{*}(f)$ is the convolution result of $s(t) e^{-j 2 \pi n f_{s} t}$ and $s^{*}(-t)$, which means that $S\left(f-n f_{s}\right) S^{*}(f)$ is the output spectrum of target echo (with Doppler frequency shift $f_{d}=-n f_{s}$ ) after matched filter. $S\left(f-n f_{s}-l / P T_{c}\right) S^{*}(f)$ means the spectrum of target echo result (with Doppler frequency shift $f_{d}=-n f_{s}-l / P T_{c}$ ) after matched filter [14].

The inverse Fourier transform of $S\left(f-n f_{s}\right)$ after matched filter is as below

$$
y_{j n}(t)=F^{-1}\left[S\left(f-n f_{S}\right) S^{*}(f)\right],
$$

where $F^{-1}$ is the inverse Fourier transform and $y_{j n}(t)$ is the output signal of matched filter. According to the theory of ambiguity function, $y_{j n}(t)$ can be written as

$$
y_{j n}(t)=\chi\left(t,-n f_{s}\right) .
$$

The matched filter result of $S\left(f-n f_{s}-l / P T_{c}\right)$ is as follows

$$
y_{j n l}(t)=\chi\left(t,-n f_{s}-l / P T_{c}\right) .
$$

From (23) and(24), the superposition of all the jamming signal result is

$$
y_{j}(t)=\sum_{n=-\infty}^{+\infty} a_{n} y_{j n}(t)+\sum_{n=-\infty}^{\infty} \sum_{\substack{l=-\infty \\ l \neq 0}}^{+\infty} b_{n l} y_{j n l}(t) .
$$

Equation (25) shows that $y_{j}(t)$ is a weighted superposition of the result when radar echo (with different Doppler frequency shift) passing through matched filter. The first item in (25) is the main false-target achieved by interruptedsampling. The second item is the dense multiple false-targets around $y_{j n}(t)$. These dense false-targets created by pseudorandom sequence are named as dense noise in the following of the article.

Next we take the main false target with Doppler frequency shift $\xi_{n}=-n f_{s}$ as an example to analyze the output of jamming signal after LFM signal pulse compression. The ambiguity of LFM signal is defined as follows [14]

$$
\begin{aligned}
\chi(\tau, \xi)= & \frac{\sin [\pi(\xi-k \tau)(T-|\tau|)]}{\pi(\xi-k \tau)(T-|\tau|)}\left(1-\frac{|\tau|}{T}\right) \\
& \exp (-j \pi \xi \tau) \quad \text { when }|\tau|<T
\end{aligned}
$$

The ambiguity of (26) is shown in Fig. 6. The time delay $\tau$ is strong correlated to the frequency shift $\xi$ in LFM signal. It means that when $\Delta \xi$ changes in frequency domain, there will be a change of $\Delta t$ in time domain. So the shift-frequency jamming on LFM signal will induce range deception jamming to radar. 


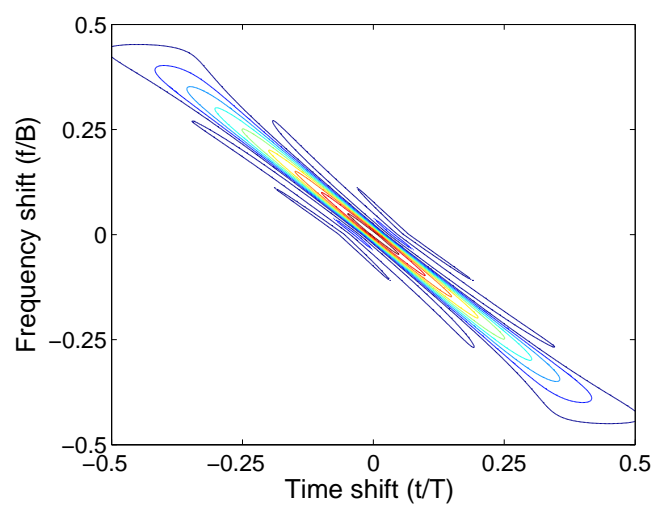

Fig. 6. Ambiguity of LFM signal.

Substituting $y_{j n}(t)$ in (26) into (23), we derive

$$
\begin{aligned}
y_{j n}(t)= & \operatorname{sa}\left[\pi\left(n f_{s}+k t\right)(T-|t|)\right]\left(1-\frac{|t|}{T}\right) \\
& \exp \left(j \pi n f_{s} t\right) \quad \text { when }|t|<T .
\end{aligned}
$$

The envelope of $y_{j n}(t)$ is shown in (28).

$$
\begin{aligned}
\left|y_{j n}(t)\right|= & \left|\operatorname{sa}\left[\pi\left(n f_{s}+k t\right)(T-|t|)\right]\left(1-\frac{|t|}{T}\right)\right| \\
& \text { when }|t|<T .
\end{aligned}
$$

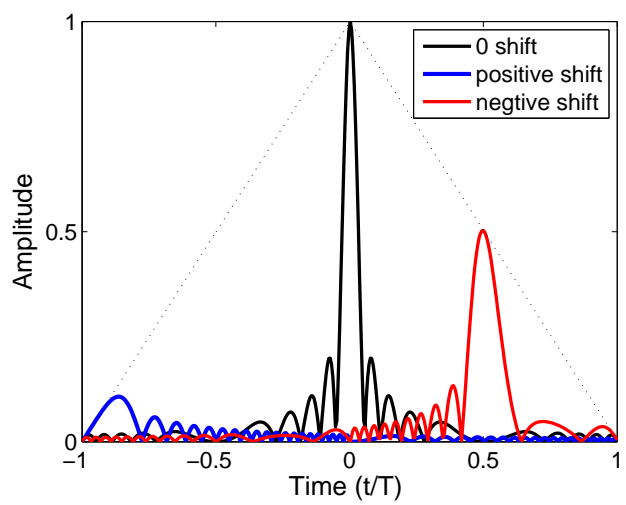

Fig. 7. Envelope of shift-frequency signal after matched filter.

Figure 7 shows the envelope $\left|y_{j n}(t)\right|$. When Doppler frequency shift $\xi_{n}=0$, the peak of pulse compression output appears in time 0 . The width of peak is $[-1 / B, 1 / B](B$ is the bandwidth of LFM signal) and the amplitude is modulated by $s a$ function. When $\xi_{n} \neq 0$, the location of peak moves to $t=-\xi_{n} / k$. The location of peak moves ahead when $\xi_{n}>0$, otherwise moves back. To sum up, the shift-frequency jamming to LFM signal is capable of creating false-target.

From (28) we can see that $\left|y_{j n}(t)\right|$ is maximized when $t_{\max }=-\xi_{n} / k$. The amplitude of this false-target is

$$
\begin{aligned}
y_{s n \max } & =\left|y_{s n}\left(t_{\max }\right)\right|=\left|1-\frac{\left|t_{\max }\right|}{T}\right| \\
& =\left|1-\frac{\left|-\xi_{n} / k\right|}{T}\right|=1-\frac{\left|\xi_{n}\right|}{B} .
\end{aligned}
$$

The time delay between the peak of real target and false target created by shift-frequency signal is

$$
\Delta t=\frac{\left|\xi_{n}\right|}{k} .
$$

From (30) we can see that when $k$ is fixed, $\Delta t$ is proportional to $\left|\xi_{n}\right|$. From theory of signal processing, the output signal is achieved when radar echo and matched filter is overlapped. When $\left|\xi_{n}\right|$ becomes large, then overlapping segment becomes small, which means that the amplitude of false-target decreases. The matched filter will not produce any output signal when $\left|\xi_{n}\right| \geq B$. So (30) must satisfy the condition $\left|\xi_{n}\right|<B$. Compared with the complete matched LFM signal, mismatch will appear when shift-frequency signal passing through the matched filter. The width of output signal's peak will spread and the amplitude will decrease according to the trigonometric function, which will impact the jamming power. Normally, the bandwidth of LFM signal is quite large and $\left|\xi_{n}\right|<B$ is easy to be satisfied. So interrupted-sampling can produce $3-5$ effective false-targets against LFM radar.

The analysis of shift-frequency signal created by pseudo-random sequence is similar to the procedure above. After jamming signal $y_{j}(t)$ passing through matched filter, the output signal is the superposition of false-targets produced by both interrupted-sampling pulse and pseudorandom sequence phase-modulation. Taking the amplitude $a_{n}$ and $b_{n l}$ in (15) into consideration, the amplitude of main false-target is quite small and sampling frequency of interrupted-sampling determines the distribution of dense noise in frequency domain. The dense noise around the 0 -level false-target contributes to the jamming effect.

\section{Parameters Discussion}

\subsection{Sampling Frequency}

The sampling frequency $f_{s}$ of interrupted-sampling determines the distribution of main false-target in range direction. When the parameters of LFM signal is fixed, distance between false-targets is proportional to $f_{s}$. Taking the Doppler frequency shift $\xi_{n}=-n f_{s}$ into consideration, the time delay between the peak of neighboring false-targets is

$$
\Delta t_{\max 1}=\frac{f_{s}}{k} .
$$

Assume that the bandwidth of LFM signal is $5 \mathrm{MHz}$ and pulse width is $100 \mu \mathrm{s}$, sampling frequency of interruptedsampling pulse is $0.25 \mathrm{MHz}$. The distance between main false-targets is $1500 \mathrm{~m}$. Under the situation when duty ratio is $50 \%$, the level of even level false-target is 0 . Thus the distance between $n$ level false-target and $n+2$ level false-target is $3000 \mathrm{~m}$ ( $n$ is odd).

The matched filter output of $n$ level shift-frequency signal is modulated by both $a_{n}$ and $\left|y_{s n}\left(t_{\max }\right)\right|$, where $a_{n}$ is determined by the characteristic of interrupted-sampling pulse and $\left|y_{s n}\left(t_{\max }\right)\right|$ is correlated to match filter. 


\subsection{Code Width}

Similar to (31), the distance between the dense noise created by pseudo-random sequence is

$$
\Delta R_{\text {noise }}=\frac{c / P T_{c}}{2 k} .
$$

The parameter of LFM is the same with that in Sec. 4.1. The period of sequence is 511 and code width is $1 \mu \mathrm{s}$. Then the corresponding distance between two noise points is $5.9 \mathrm{~m}$.

The amplitude of $n l^{\text {th }}$ level dense noise after matched filter is determined by $b_{n l}$ and $\left|y_{s n}\left(t_{\max }\right)\right| . b_{n l}$ is the amplitude characteristic of interrupted-sampling pulse and pseudorandom sequence. $b_{n l}$ is 0 every $P$ numbers of code width. According to the analysis in Sec. 2.2, the dense noise created by pseudo-random sequence covers a distance span of $6000 \mathrm{~m}$.

From the analysis above we know that the main jamming power is around the 0 level main false-target, which locates close to the real target. The dense noise around 0 level false-target gets the main jamming power and covers our protected target.

The analysis in Sec. 2.2 suggests that different $T_{c}$ has different modulation bandwidth, so is there an optimal $T_{c}$ to achieve the best jamming effect? Next we demonstrate some conditions to seek the optimal value.

First and foremost, we aim at creating noise depressing jamming to victim radar, thus the distance between two false targets should be less than the range resolution of radar, of which the ideal value is $c / 2 B$ and $B$ stands for the bandwidth of radar signal. Thus we have condition one

$$
\frac{c / P T_{c}}{2 k} \leq \frac{c}{2 B} \text {. }
$$

After a few steps we derive

$$
T_{c} \geq \frac{B}{P k} .
$$

There is one more point, according to the travel of pseudo-random sequence that the maximum number of continuous codes with the same value is $n-1$, where $n$ is the level of sequence. So we need the value of the sequence at least change one time during the transmitting window of interrupted-sampling to make sure that the phase-modulation process is conducted on the sampled radar signal. If the modulation process does not happen, the transmitted jamming signal will be part of the original radar signal and it may increase the target echo, which is not good for jamming. Therefor at least $n$ codes are wanted during one segment of jamming signal, that is

$$
T_{c} \leq \frac{T_{s}}{2 n} .
$$

The last but not the least, modern radar uses constant false alarm rate (CFAR) detection to seek the target. The purpose of jamming signal in CFAR detection is to create large quantities of noise, which will enhance the threshold of detection and thus the target echo is beneath the threshold. So the covering distance of noise should be equivalent or larger than the detection window of CFAR detection. For example, if CFAR uses $N$ units to conduct average computation and the resolution of this radar is $\Delta r$, then the covering distance of jamming signal(it can be computed according to (32)) and the detection window of CFAR should satisfy

$$
2 \times \frac{c / T_{c}}{2 k} \geq \Delta r \times N .
$$

If both the three conditions above can be satisfied, we derive a range for $T_{c}$ and the jamming signal based on these values shall be effective to hostile radar. Otherwise, when three conditions conflict with each other, we think (35) should be meet above all.

\section{Simulation Results}

This section presents the simulation results of jamming effect to wideband radar. The bandwidth of LFM is $5 \mathrm{MHz}$ and pulse width is $100 \mu \mathrm{s}$. The sampling period of interrupted-sampling is $8 \mu \mathrm{s}$. The jammer and target with $R C S=1 \mathrm{~m}^{2}$ are located $15 \mathrm{~km}$ away from radar. Figure 8 shows the pulse compression result of target echo, jamming signal and radio frequency (RF) noise. The amplitudes of jamming signal and RF noise are normalized by the amplitude of target echo.

From Fig. 8 we can see the peak of jamming signal is behind the real target. This result is caused by the $50 \%$ duty ratio of interrupted-sampling pulse. Under this circumstance, the sampling window and transmitting window is $4 \mu$ s respectively and the peak of jamming is fall behind. This hysteretic $4 \mu$ s corresponds to $1.2 \mathrm{~km}$ in range direction, which is the location of 0-level main target created by interrupted-sampling. The dense noise of pseudo-random sequence is symmetrically distributed around 0-level main target. The primary covering effect to the real target is produced by the noise points of frequency lying in $\left[-1 / T_{c}, 1 / T_{c}\right]$, which occupies most power of jamming signal. The main jamming effect is achieved by the dense noise around 0-level false-target. Other false-target and noise with large value of shift-frequency can be neglected for the lower amplitude reasons.

Fig. 9 shows the result of pulse accumulation of target echo, jamming signal and RF noise. By pulse accumulation, radar enhances the signal-to-noise ratio significantly. Because of the non-coherence with radar signal, RF noise can't obtain processing gain while the amplitude of our jamming signal still rises after pulse accumulation.

As is shown in Fig. 10, when $T_{c}$ is small, jamming signal can produce a quite wide covering span but the amplitude is small. When $T_{c}$ becomes large, a narrow covering span of large jamming amplitude will be achieved. So we should 


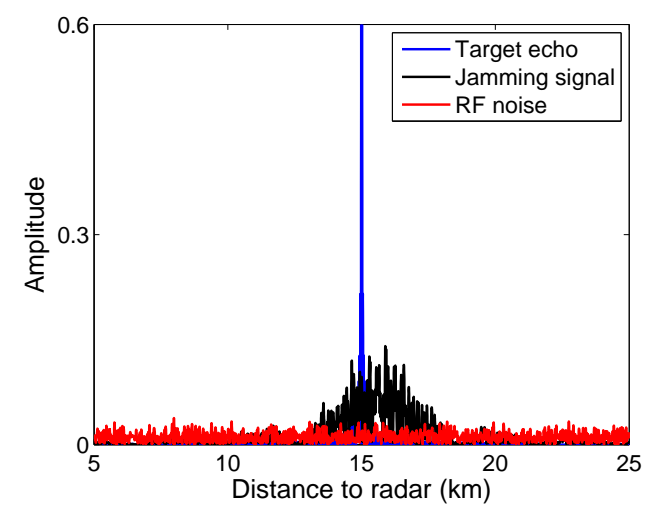

Fig. 8. Pulse compression result when $T_{c}$ is $1 \mu \mathrm{s}$.

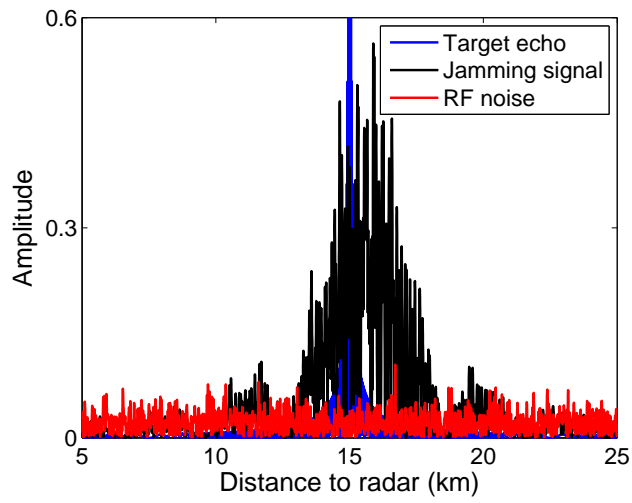

Fig. 9. Pulse accumulation results.

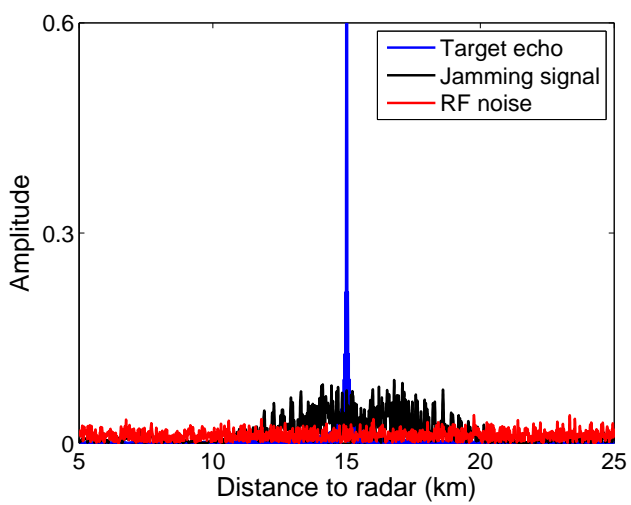

Fig. 10. Pulse compression result when $T_{c}$ is $0.5 \mu \mathrm{s}$.

take the jamming power into consideration when selecting the code width $T_{c}$. The short $T_{c}$ is preferred to guarantee a large covering distance. Meanwhile $T_{c}$ can't be too small for the decreasing effect in amplitude of jamming signal.

As a comparison, interrupted-sampling repeater jamming (ISRJ) is shown in Fig. 11. The coherence of ISRJ with the matched filter is higher than our proposed jamming signal and the amplitude of main false target is almost 50\% of target echo. But from Fig. 11 we also see that at most 5 false targets are achieved and they can't destroy the location of target even when the jamming power is large. The finite false target is unable to enhance the CFAR threshold.

Figure 12 manifests shift frequency jamming modulated by sawtooth wave. Through the frequency modulation by pe-

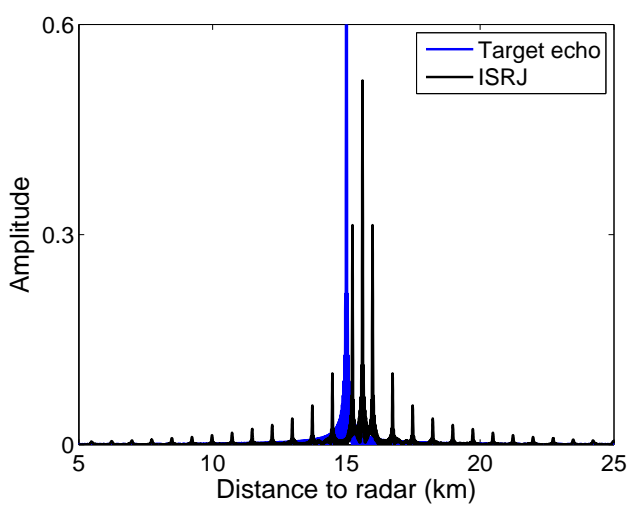

Fig. 11. PC result of interrupted-sampling repeater jamming.

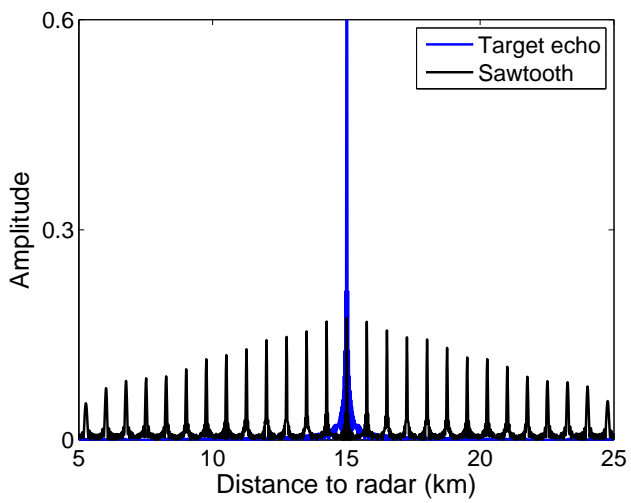

Fig. 12. PC result of shift frequency jamming.

riodic sawtooth wave, the false targets with the same spacing are achieved, of which the amplitude decreases because the mismatch with matched filter. Although more false targets are induced after pulse compression compared with ISRJ, they are still not dense enough to smear the target echo. And the false target far away from the target hardly contributes to the jamming effect. As is introduced in Sec. 1, frequency modulation requires structure of high complexity and consumes more resources. Therefore in terms of seeking a way of producing effective noise jamming, our method is more convenient and impactful.

Next we analyze the CFAR detection results of different jamming signals. The false alarm rate is set as $10^{-6}$, protection unit is 3 and averaging unit is 50 in each side. Simulation analyzes the lowest needed jamming power when real target can't be distinguished.

Figures 13 and 14 present the CFAR results of different jamming parameters. Figures 13 and 14 need a jammingto-signal ratio (JSR) of $9.0 \mathrm{~dB}$ and $9.5 \mathrm{~dB}$ respectively. The dense noise covers some range cells and enhances the CFAR threshold. The real target is below the dense noise and it can't be detected. Considering that CFAR uses multiple average cells to derive the threshold, the dense noise should cover as much range cells as possible when the jamming amplitude is enough. Figure 14 shows the jamming result of a wide $T_{c}$. On this occasion, the large amplitude noise is achieved and some noise raises the amplitude of target. Because this noise doesn't cover a long range cell, it needs more jamming power 


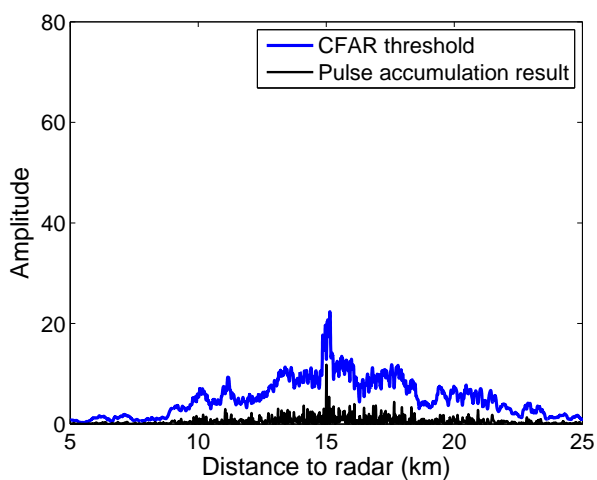

Fig. 13. $T_{c}=0.2 \mu \mathrm{s}$

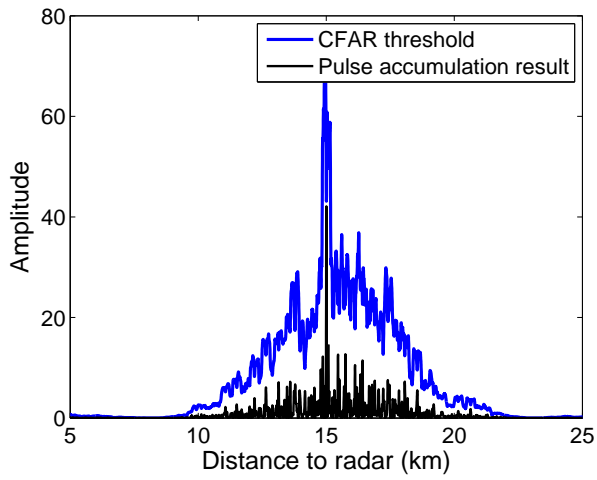

Fig. 14. $T_{c}=0.4 \mu \mathrm{s}$.

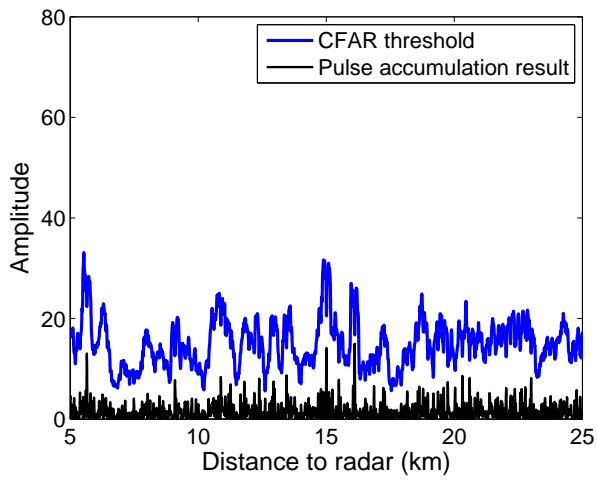

Fig. 15. CFAR result of RF noise.

compared with Fig. 13.

Figure 15 demonstrates the CFAR detection result of RF noise. The noise signal is everywhere along the range direction and with a quite constant amplitude. Because this RF noise is non-coherent with the reference signal of matched filter, it needs more jamming power to decrease the SNR in radar receiver. To overlap the real target, RF noise needs a JSR of $33.6 \mathrm{~dB}$, which is significantly larger than our jamming signal.

\section{Implementation}

The jamming signal producing module consists of AD converter, low pass filter, FPGA and DA converter. After detected by jammer, radar signal is down-converted, through low-pass filter and sent to AD converter. FPGA fulfills the storing procedure of quantified radar signal and the modulation of jamming signal.

ADC083000 is chosen as the AD converter, which operates at the sampling frequency of $2.4 \mathrm{GHz}$ and quantified data is output in 4 channels. The sampled signal can be selected in the expression of 8 bit unsigned data. Based on the analysis in previous section, the pseudo-random sequence is created by a 9-bit shift-register at $50 \mathrm{MHz}$ clock. At the transmitting stage, the jamming signal is modulated by the value of pseudo-random sequence. Because the value of sequence is +1 or -1 , the phase modulation of jamming signal is quite simple in this situation. When the value is +1 , the jamming signal equals to the original sampled radar signal, otherwise is the opposite value of radar signal.

After the creation procedure, jamming signal is emitted at the transmitting stage of the jammer. AD9739 is chosen as the DA converter. The data transmission in FPGA is shown is Fig. 16. Because AD9739 adopts 2 channels for inputting digital data, the stored radar signal needs to be re-arranged before DA converted. AD9739 uses 14-bit to quantify the digital input signal, thus the 8-bit sampled radar signal should be amplified to reach 14-bit. In order to maximize the jamming power, the output jamming signal should reach the maximum amplitude output ability of DA converter. When the amplitude of sampled radar signal is small, the jamming signal is multiplied by a big coefficient, otherwise by a small coefficient.

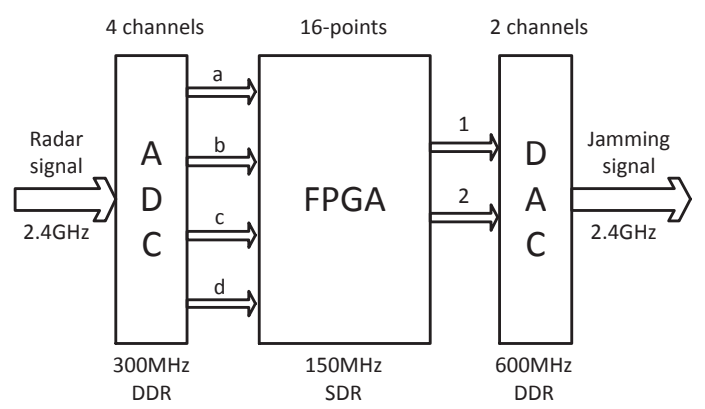

Fig. 16. Data transmission in FPGA.

When the jammer selects a high sampling frequency, the DDR data rate of DA converter is also high. Taking the $2.4 \mathrm{GHz}$ sampling frequency for example, DA converter receiver channels operate at $600 \mathrm{MHz}$ DDR mode, which is hard to be satisfied in FPGA logic resources. Thus the output data for DA converting can be processed by the Select_IO resource in Xilinx Virtex5 serial (or advanced serial) FPGA. FPGA works at the $150 \mathrm{MHz}$ clock, which means 16 sampling points are stored at one clock period. These 16 points need to be re-arranged to satisfy the 2 receiver channels in DA converter. So we firstly divide 16 points into two groups according to the odd, even numbers.

The group made up of odd number sampling points is used to introduce the re-arrange process. OSERDES is used for converting parallel data to serial data and the re-arrange 
method for these points is shown in Fig. 17. The new data is created by the combination of the same bit in 8 sampling points. For example, vector 1 is made up of the MSB of each sampling point. The re-arranged data is sent to OSERDES for further process. Because DA converter takes up 14-bit, 14 OSERDES are needed. The processing procedure of OSERDES is shown in Fig. 18. Vector 1 is the parallel data in $150 \mathrm{MHz}$ SDR mode and the output serial signal is in $600 \mathrm{MHz}$ DDR mode. And 14 Oserders work together to fulfill the 8 sampling points transmission to DA converter.

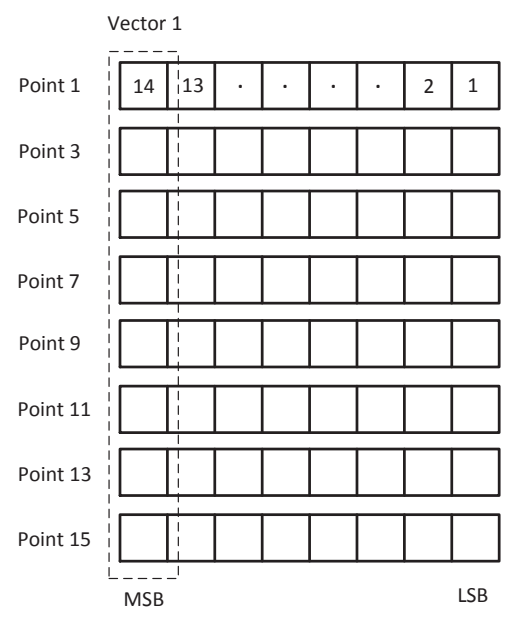

Fig. 17. Data re-arrange process.

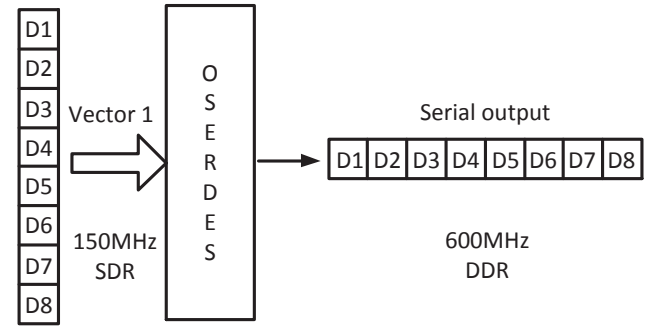

Fig. 18. OSERDES.

The spectrums of LFM signal (upper) and corresponding jamming signal (under) are shown in Fig. 19, which is the same with the analysis in Sec. 2.3. The bandwidth of LFM signal is $10 \mathrm{MHz}$, and the jamming signal has a wider spectrum width. From the figure we see that the amplitude of jamming signal decreases compared with radar signal. This power loss can be compensated by the radio frequency power amplify. The spectrum of jamming signal corresponds to the analysis in above section.

If radar transmits different radar signal, jammer can produce the jamming signal which is similar to the original signal but with a wider spectrum width. Suppose that radar transmits sine signal, the spectrums of sine signal (upper) and jamming signal (under) are shown in Fig. 20. From the figure we see that a spread spectrum is achieved and the center frequency of jamming signal is coherent with the sampled radar signal. Thus the jammer based on our proposed jamming method is adaptive to different types of radar signal and can produce a partial coherent noise jamming.

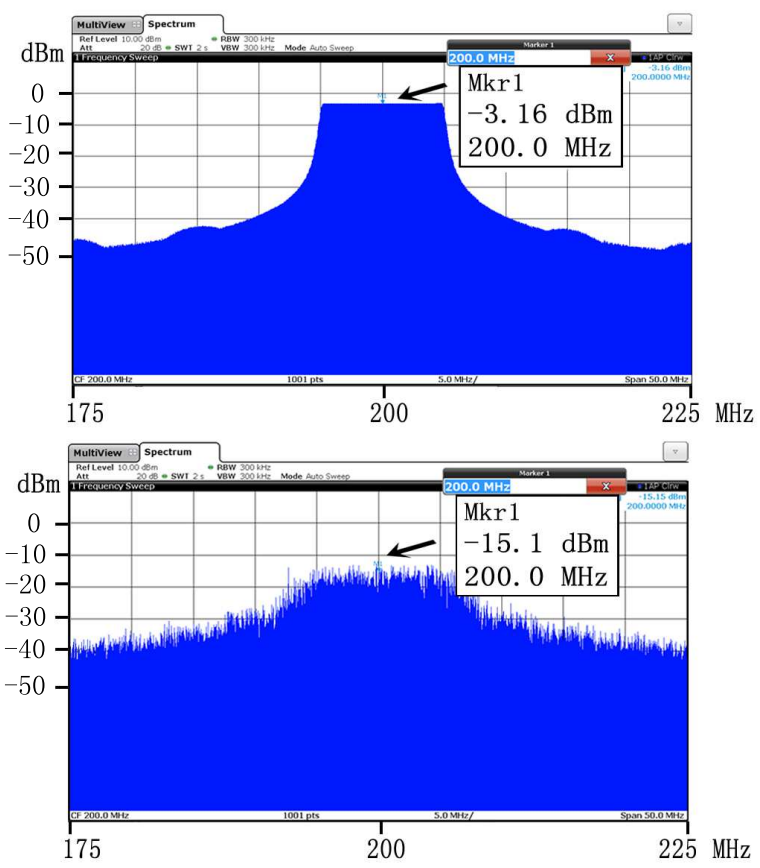

Fig. 19. Spectrums of LFM signal and corresponding jamming signal.

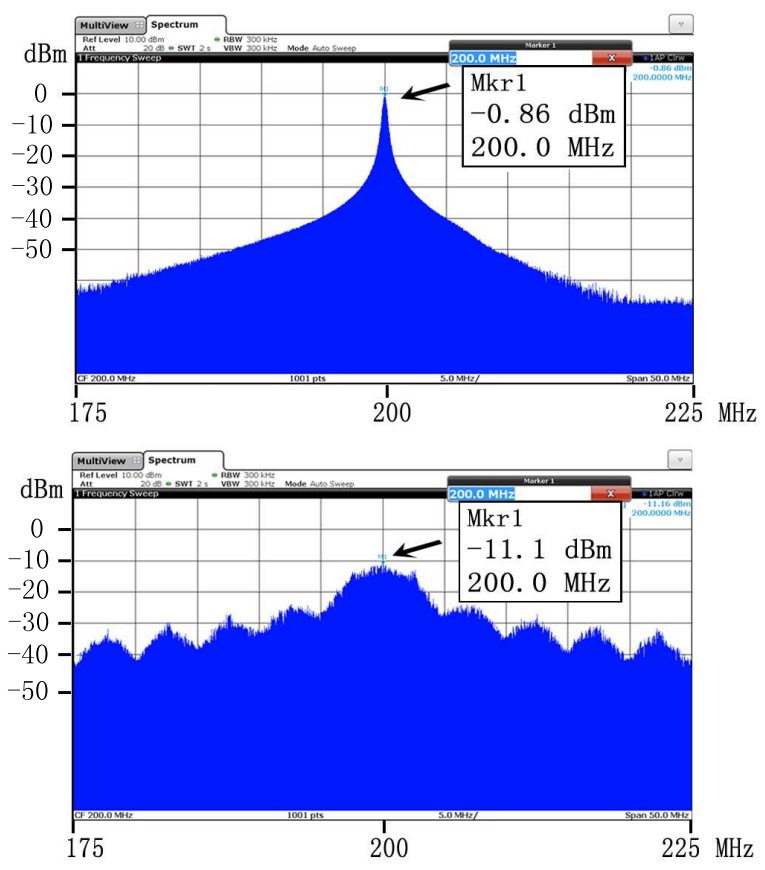

Fig. 20. Spectrums of sine signal and corresponding jamming signal.

\section{Conclusion}

Aim at the current situation that non-coherent jammer needs large jamming power, we propose a quasi-coherent jamming method based on interrupted-sampling and pseudorandom sequence phase-modulation. The merit and implementation produce of this jamming signal is analyzed in detail. By interrupted-sampling, jammer can react quickly to 
radar signal and produce jamming signal. The dense noise created by pseudo-random sequence phase-modulation covers some frequency span and protects the real target effectively. Simulation results validate the correctness and effectiveness of the proposed jamming signal. Compared with other non-coherent noise signal, this jamming signal is partial coherent with radar signal. It obtains some processing gain after pulse compression and jams victim radar effectively with lower jamming power. This quasi-coherent jamming signal is easy to be implemented and doesn't need complex phase-modulation. It is with strong adaptability to radar signal and adapts to variable radar signal. Different jamming effect will be achieved by flexibly controlling jamming parameters.

The main purpose of this jammer is to produce a coherent noise jamming that covers target echo after pulse compression. Compared with frequency modulated noise, the structure of our jammer is less complex, which needs one channel of radar signal. From my own perspective, the phase of radar signal is the foundation of frequency modulation, which needs quadrature demodulation. And this increases the difficulty and complexity of jammer. Before transmitting the frequency modulated jamming signal, the full radar signal shall be intercepted to produce jamming signal, which causes jamming signal to lag behind radar signal at least one pulse.

Furthermore, thanks to the property of pseudo-random sequence, +1 and -1 are the only two values of the sequence, the phase-modulation becomes quite simple. As an example, on Xilinx FPGA we can use Slice resource to complete the modulation process, according to the analysis in Sec. 2.3. Convolution modulation contains multiplication and addition, which needs the precious multiplier resource to achieve correct result when the chip is working on high frequency. In addition, FPGA with more multipliers, high performance, of course high price, will increase the design cost.

The jammer of the proposed method brings capability of self-defense to our important target, such as airplane. When fixed on the airplane, the jammer transmits jamming signal when detecting radar signal, which covers the real target echo. An ocean of noise appears on the radar receiver and the real characteristic of airplane is smeared. This noise signal will prevent hostile radar from detecting our target and thus the survivability of target is enhanced. And this jammer also brings a solution to ground target to resist the detection of airborne radar, such as SAR. Through the movement of radar platform, SAR obtains high-resolution image of its interesting area. From the analysis above we know that the jammer produces a covering distance in range-direction. Thus more jammer located around our ground target in different azimuth-direction can be joined together to produce a covering region. So the feature of our target in this region can be submerged under the noise protection. To sum up, our jamming method is of some meaning to jamming signal design and engineering implementation.

\section{Acknowledgments}

This work was supported in part by the National Natural Science Foundation of China under Grant no. 61302017. The authors would like to thank the anonymous reviewers for the improvement of this paper.

\section{References}

[1] MOHAMMADPOOR, M., ABDULLAH, R., ISMAIL, A., et al. A linear frequency modulated bistatic radar for on-the-ground object detection. In Proceedings of 2011 IEEE CIE International Conference on Radar Chengdu (China), 2011, p. 63-66. DOI: 10.1109/CIERadar.2011.6159476

[2] PAN, X. Y., WANG, W., FENG, D. J., et al. On deception jamming for countering bistatic ISAR based on sub-Nyquist sampling. IET Radar, Sonar \& Navigation, 2014, vol. 8, no. 3, p. 173-179. DOI:10.1049/iet-rsn.2013.0020

[3] YEH, C. M., YANG, J., SHAN, X. M., et al. Simultaneous range and radial velocity estimation with a single narrowband LFM pulse. Journal of Systems Engineering and Electronics, 2012, vol. 23, no. 3, p. 372-377. DOI: 10.1109/JSEE.2012.00046

[4] SHABANI, M., AKBARI, M. Non-linear effects of intensitymodulated and directly detected optical links on receiving a linear frequency-modulated waveform. IET Optoelectronics, 2011, vol. 5, no. 6, p. 255-260. DOI: 10.1049/iet-opt.2010.0082

[5] OIIVIER, K., CILlIERS, J. E., PLESSIS, M. Design and performance of wideband DRFM for radar test and evaluation. Electronics Letters, 2011, vol. 47, no. 14, p. 824-825. DOI: 10.1049/el.2011.0362

[6] TANG, P. F., GUO, S. J., CHEN, Z. P. Design and simulation of DRFM system based on digital channelized receiver and transmitter In Proceedings of IEEE International Conference on Mechatronic Science, Electric Engineering and Computer (MEC). Jilin (China), 2011, p. 270-273. DOI: 10.1109/MEC.2011.6025453

[7] LI, Q. H., YANG, D., MU, X. H., et al. Design of the L-band wideband LFM signal generator based on DDS and frequency multiplication. In Proceedings of IEEE International Conference on Microwave and Millimeter Wave Technology (ICMMT). Shenzhen (China), 2012, p. 1-4. DOI: 10.1109/ICMMT.2012.6230321

[8] YANG, Y., ZHANG, W. M., YANG, J. C. Study on frequency-shifting jamming to linear frequency modulation pulse compression radar. Proceedings of International Conference on Wireless Communications \& Signal Processing (WCSP). Nanjing (China), 2009, p. 1-5. DOI: $10.1109 /$ WCSP.2009.5371387

[9] FITZGERLD, R. J. Effect of range-Doppler coupling on chirp radar tracking accuracy. IEEE Transactions on Aerospace and Electronic Systems, 1974, vol. 10, no. 4, p. 528-532. DOI: 10.1109/TAES.1974.307809

[10] CAI, X. F. SONG, J. S. ZHANG, X. M. et al. A jamming technique against SAR based on inter-pulse subsection randomly-shiftfrequency and its application. In Proceedings of IEEE China Summit \& International Conference on Signal and Information Processing (ChinaSIP). Xi' an (China), 2014, p. 785-789. DOI: 10.1109/ChinaSIP.2014.6889352

[11] WANG, Y. J., ZHAO, G.Q., HU, X.M. Method of shift-frequency Jamming to LFM Radar Based on Delay Invariance. Systems Engineering and Electronics, 2009, vol. 31, no. 8, p. 1861-1863.(in Chinese). DOI: 10.3321/j.issn:1001-506X.2009.08.018 
[12] WANG, Y. J., ZHAO, G. Q. Blind Nth-order SSC shift-frequency jamming to LFM radar (in Chinese). Journal of Circuits and Systems, 2011, vol. 16 , no. 4, p. 70-74. DOI: 10.3969/j.issn.10070249.2011.04.014

[13] FENG, D. J., TAO, H. M., YANG, Y. Jamming de-chirping radar using interrupted-sampling repeater. Science China Information Sciences, 2011, vol. 54, no. 10, p. 2138-2146. DOI: 10.1007/s11432011-4431-4

[14] WANG, X. S., LIU, J. C., ZHANG, W. M., et al. Mathematic principles of interrupted-sampling repeater jamming (ISRJ). Science in China Series F: Information Sciences, 2007, vol. 50, no. 1, p. 113-123. DOI: 10.1007/s11432-007-2017-y

[15] LIU, Z., WANG, X. S., LIU, J. C. Jamming technique of interruptedsampling and periodic repeater based on digital radio frequency memory (in Chinese). Acta Armamentaii, 2008, vol. 29, no. 5, p. 405-410. DOI: $10.3321 /$ j.issn:1000-1093.2008.04.005

[16] FENG, D. J., XU, L. T., WANG, W., et al. Radar echo cancellation using interrupted-sampling repeater. IEICE Electronics Letters, 2014, vol. 11, no. 8, p. 1-6. DOI: 10.1587/elex.11.20130997

[17] XU, L. T., FENG, D. J., LIU, Y. C., et al. A three-stage active cancellation method against synthetic aperture radar. IEEE Sensors Journal, 2015, vol. 15, no. 11, p. 6173-6178. DOI: 10.1109/JSEN.2015.2453396

[18] WANG, W. Q., CAI, J. Y. A technique for jamming bi- and multistatic SAR systems. IEEE Geoscience and Remote Sensing Letters, 2007, vol. 4, no. 1, p. 80-82. DOI: 10.1109/LGRS.2006.886036

[19] PACE, P. E., FOUTS, D. J., EKESTORM, S., et al. Digital falsetarget image synthesiser for countering ISAR. IEE Proceedings Radar Sonar Navigation, 2002, vol. 149, no. 5, p. 248-257. DOI: 10.1049/iprsn:20020635

[20] FOUTS, D. J., PACE, P. E., KAROW, C., et al. A single-chip false target radar image generator for countering wideband imaging radars. IEEE Journal of Solid-State Circuits, 2002, vol. 37, no. 6, p. 751-759. DOI: 10.1109/JSSC.2002.1004579

[21] XU, L. T., FENG, D. J., PAN, X. Y., et al. An improved digital false-target image synthesizer method for countering inverse synthetic aperture radar. IEEE Sensors Journal, 2015, vol. 15, no. 10, p. 5870-5877. DOI: 10.1109/JSEN.2015.2453163
[22] ZHAO, B., ZHOU, F., SHI, X. R., et al. Multiple targets deception jamming against ISAR using electromagnetic properties. IEEE Sensors Journal, 2015, vol. 15, no. 4, p. 2031-2038. DOI: 10.1109/JSEN.2014.2368985

[23] GONG, S. X., WEI, X. Z. Noise jamming to ISAR based on multiplacation modulation. In Proceedings of 2013 IET International Radar Conference. Xi' an(China), 2013, p. 1-5. DOI: 10.1049/cp.2013.0398

[24] GONG, S. X., WEI, X. Z., LI, X., et al. Mathematic principle of active jamming against wideband LFM radar. Journal of Systems Engineering and Electronics, 2015, vol. 26, no. 1, p. 50-60. ISSN: 1004-4132. DOI:10.1109/JSEE.2015.00008

[25] ZHANG, Y. B. Technology of smart noise jamming based on multi-plication modulation (in Chinese). In Proceedings of International Conference on Electric Information and Control Engineering(ICEICE). Wuhan(China), 2011, p. 4557-4559. DOI: 10.1109/ICEICE.2011.5777633

[26] GUO, J. J., WANG, X. H., WANG, X., et al. New smart noise jamming of radar signal frequency modulation (in Chinese). Journal of Xidian University, 2013, vol. 40, no. 4, p. 155-160. DOI:10.1109/JSEE.2015.00008

\section{About the Authors...}

Ning TAI was born in 1989. He received his M.Sc. from National University of Defense Technology in 2013. His research interests include radar signal processing and radar system simulation.

Yujian PAN was born in 1987. He received his M.Sc. from National University of Defense Technology in 2012. His research interests include array signal processing and microwave circuit design.

Naichang YUAN was born in 1965. He received his M.Sc. and Ph.D. from Electronic Science and Technology from University of Electronic Science and Technology of China in 1991 and 1994, respectively. His research interests include array signal processing, SAR imaging processing and signal processing in radar. 